\title{
Aggressive angiomyxoma of the thigh: A case report and review of the literature
}

\author{
XINYING LIU ${ }^{1}$, XINGHUA $\mathrm{LI}^{2}$ and YINLONG YANG ${ }^{3}$ \\ Departments of ${ }^{1}$ Ultrasound, ${ }^{2}$ Urinary Surgery, and ${ }^{3}$ Surgical Oncology, \\ The First Affiliated Hospital of Wenzhou Medical College, Wenzhou, Zhejiang 325000, P.R. China
}

Received February 15, 2012; Accepted May 21, 2012

DOI: $10.3892 / \mathrm{ol} .2012 .743$

\begin{abstract}
In this study, we report a case of aggressive angiomyxoma (AAM) of the thigh in a 43-year-old female patient. Ultrasonography revealed a $6.1 \times 3.7 \times 5.3 \mathrm{~cm}$ solid mass in the right thigh with well-demarcated borders and a heterogeneous echotexture. Color Doppler ultrasonography (CDUS) revealed weak blood flow in the mass with peripheral and central avascularity. Computed tomography (CT) of the thigh revealed a homogeneous low-density mass under the spatium intermusculare of the quadriceps femoris. The mass was diagnosed as AAM by histopathological examination following complete excision around the adventitia of the tumor. At 18 months follow-up, there was no recurrence. In conclusion, this case is noteworthy as it involves AAM of the thigh, which is a rare tumor.
\end{abstract}

\section{Introduction}

Aggressive angiomyxoma (AAM) is a rare mesenchymal tumor typically occurring in the pelvis and perineum of reproductive females (1). AAM has also been described in the male larynx, genital tract and supraclavicular fossa (2-4). A notable case of AAM on the upper, lateral aspect of the right thigh in a male patient has also been reported (5). Due to the high recurrence rate, tumor excision with wide tumor-free margins should be performed for AAM. Studies suggest that hormonal manipulation may also be of value $(6,7)$. In this study, we present a case of AAM of the thigh in a 43-year-

Correspondence to: Dr Xinghua Li, Department of Urinary Surgery, The First Affiliated Hospital of Wenzhou Medical College, Wenzhou, Zhejiang 325000, P.R. China

E-mail: lixinghua1976@126.com

Abbreviations: AAM, aggressive angiomyxoma; CDUS, color Doppler ultrasonography; CT, computed tomography; PSV, peak systolic velocity; RI, resistive index

Key words: aggressive angiomyxoma, thigh, ultrasonography, computed tomography, surgery old female patient. Informed consent was obtained from the patient prior to the study.

\section{Case report}

A 43-year-old female patient presented with a solid mass on the upper, lateral aspect of her right thigh. The mass had been present for one month and had grown progressively during that period. For the previous 2 months the patient had experienced slight pain in the right thigh, which did not impact movement. She had no history of surgical trauma or pain in the right thigh.

Sonography revealed a mass of approximately $6.1 \times 3.7 \times 5.3 \mathrm{~cm}$ overlying the upper part of the right thigh, close to the inguinal region under the muscle. The mass was oval and well-demarcated with a heterogeneous echotexture (Fig. 1A). Color Doppler ultrasonography (CDUS) revealed weak blood flow in the mass with peripheral and central avascularity (Fig. 1B). A branch of the femoral artery passing alongside and attaching to the surface of the mass was evident (Fig. 1C). The maximum peak systolic velocity (PSV) and resistive index (RI) values of the artery around the mass were $48.5 \mathrm{~cm} / \mathrm{sec}$ and 0.82 , respectively (Fig. 1D).

Computed tomography (CT) showed the mass to be located on the right thigh under the spatium intermusculare of the quadriceps femoris and demonstrated a well-defined margin of hypodensity (Fig. 2). The articulation bones of the hip had normal form and homogeneous density. There was no evident destruction, absorption or hyperplasia in the bones. The articular cavity of the hip was visible and had no hydrops.

During surgery, a mass of approximately $6 \times 7 \mathrm{~cm}$ was revealed beneath the spatium intermusculare of the quadriceps femoris on the upper part of the right thigh. The mass had a moderate degree of hardness, complete adventitia and a rough surface. A marginal surgical excision was performed around the adventitia of the tumor.

Histologically, the tumor consisted of a sparse population of stellate and spindle-shaped tumor cells in the myxoid stroma intermingled with an abundance of variably dilated blood vessels (Fig. 3). Immunohistochemically, the tumor cells were positive for vimentin, CD-34 and $\alpha$-smooth muscle actin, and negative for S-100 protein, desmin and 
A

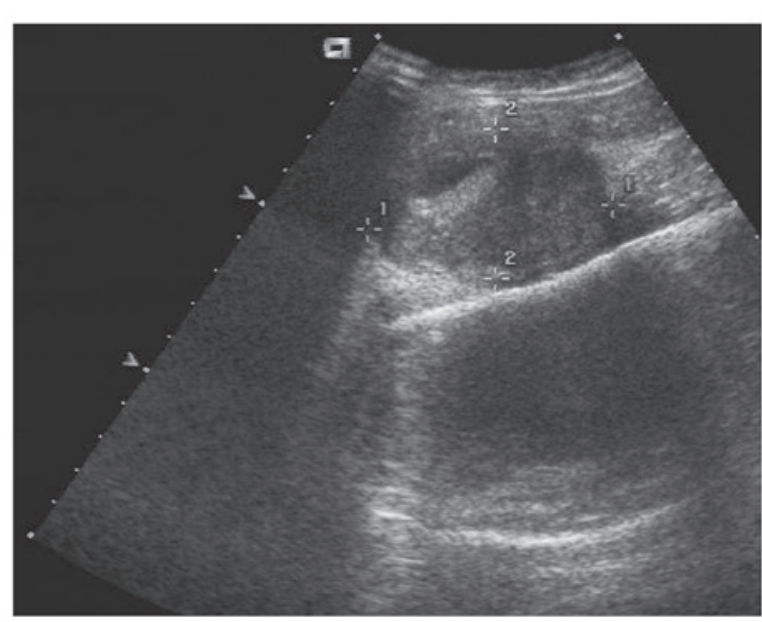

C

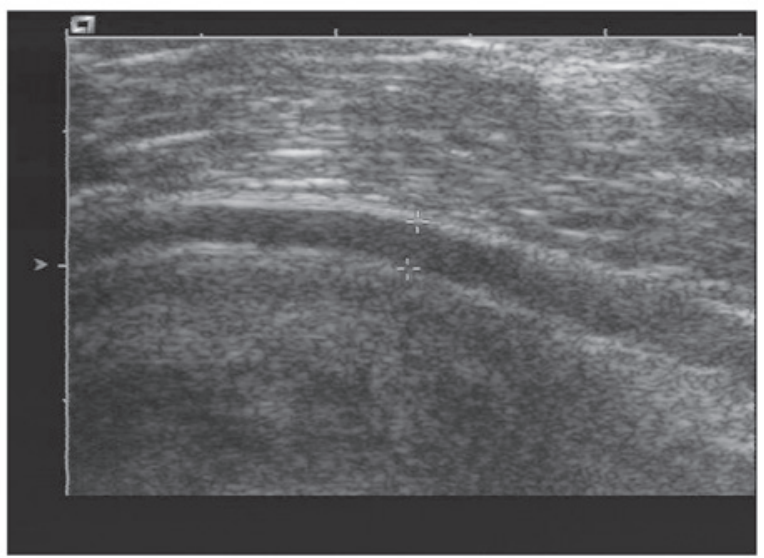

B

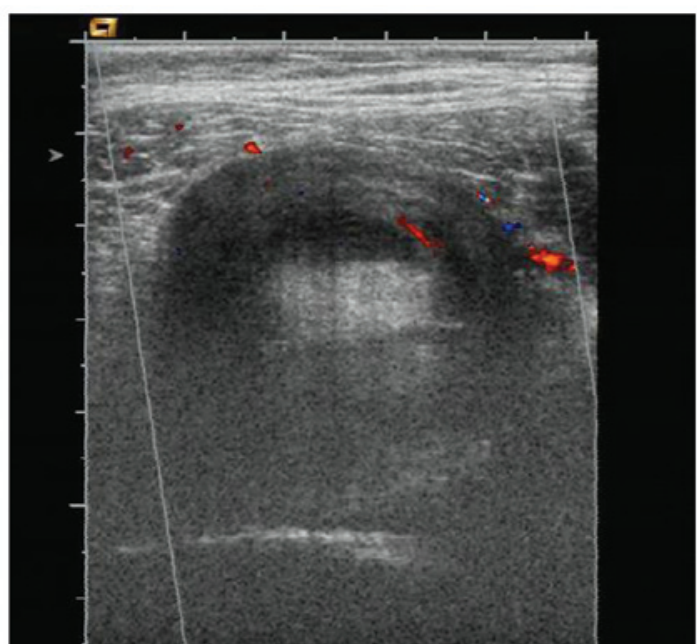

D

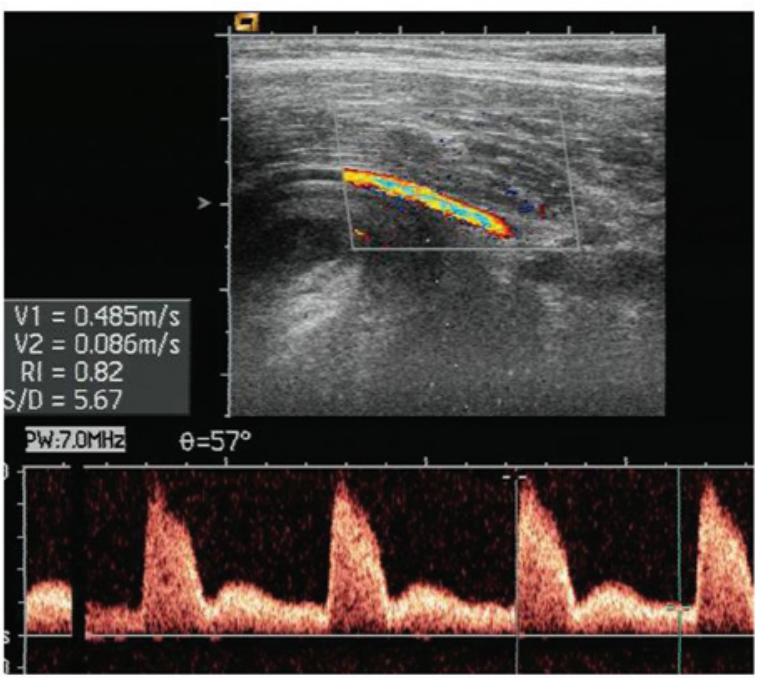

Figure 1. Sonography of aggressive angiomyxoma of the thigh. (A) The sonogram revealed a well-demarcated mass with a heterogeneous echotexture. (B) Color Doppler ultrasonography (CDUS) revealed weak blood flow in the mass as well as peripheral and central avascularity. (C) A branch of the femoral artery passing alongside and attaching to the surface of the mass can be observed. (D) The maximum peak systolic velocity (PSV) and resistive index (RI) values of the artery around the mass were $48.5 \mathrm{~cm} / \mathrm{sec}$ and 0.82 , respectively.

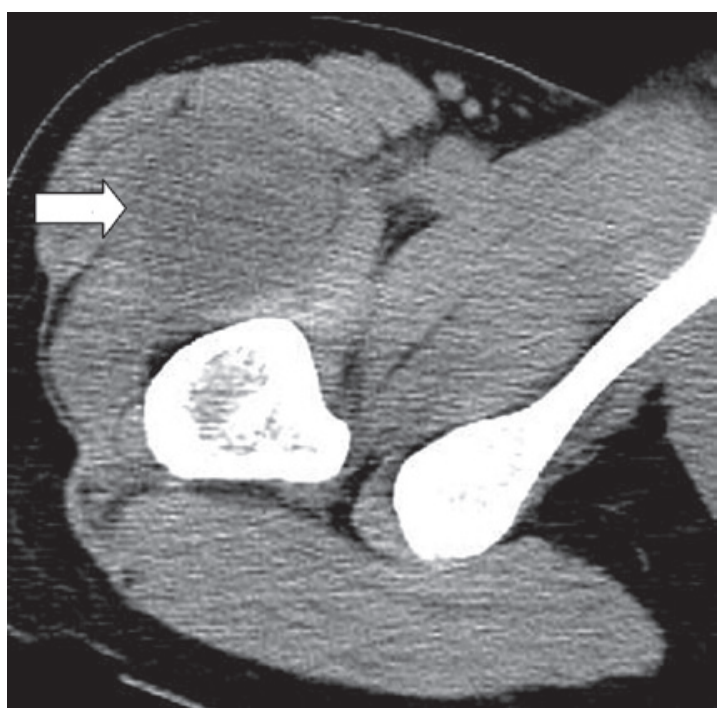

Figure 2. Computed tomography scan at the level of the right hip showing a homogeneous low-density mass (arrow) under the spatium intermusculare of the quadriceps femoris.

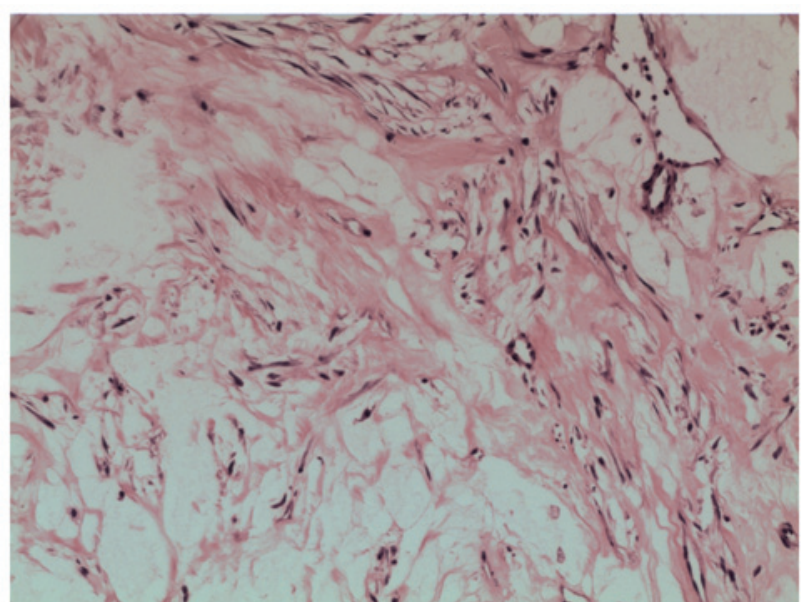

Figure 3. Photomicrograph showing a sparse population of stellate and spindle-shaped tumor cells in myxoid stroma intermingled with an abundance of variably dilated blood vessels (H\&E; magnification, x100). 
muscle-specific actin. At 18 months follow-up, there was no recurrence.

\section{Discussion}

AAM is a rare benign mesenchymal tumor which, in approximately $95 \%$ of cases, occurs in females of reproductive age (8). As reported, the female to male ratio is approximately 6.6:1 (9). AAM patients are usually asymptomatic due to the slow and insidious growth pattern of the tumor (8). Steeper and Rosai (1) described the locally aggressive behavior of the tumor and its tendency to recur locally. Surgery is the most effective method for the treatment of AAM. In contrast to other deep-seated soft tissue tumors, excision with wide tumor-free margins should be performed for AAM, rather than local excision. In addition, adjuvant therapy is necessary. Angiographic embolization may shrink the tumor preoperatively, allowing the tumor to be completely removed (10). Hormonal manipulation may be used in recurrent cases or residual tumors (11). Due to the specificity of treatment in AAM, accurate diagnosis prior to surgery is critical.

The characteristic sonographic appearance in the present case was a mass with sharply demarcated borders and a heterogeneous echotexture. In a review of the literature by Jingping et al (11), the characteristic appearance using B-ultrasound imaging for the diagnosis of AAM was described as a hypoechoic, well-demarcated mass with multiple thin, echogenic internal septa. The sonographic findings in the current study were similar to those described by Jingping et $a l$. To the best of our knowledge, the issue of blood supply in AAM presenting as a thigh tumor has never been described in detail. Heffernan et al (5) reported a case in which some internal blood flow was observed on CDUS. In our case, the tumor demonstrated weak blood flow on CDUS. AAM is derived from myofibroblasts as a phenotypic variant of the basic fibroblast (12). Furthermore, there is no evidence of an anastomosing and arborizing vascular pattern in AAM $(8,13)$. Therefore, the histological features of AAM are responsible for the reduced blood supply observed on CDUS. A branch of the femoral artery passing alongside and attaching to the surface of the mass showed low velocity and high resistance, which may be associated with the pressurization of the tumor. However, it is important to preserve the blood vessels during tumor excision due to the branch of the femoral artery beside the mass.

A CT scan was also performed prior to surgery to show the severity of the tumor. The appearance of AAM is often hypodense on CT (14). In the present study, the mass was hypodense with no invasion of the skeletal structures and no hydrops in the articular cavity. Enhanced CT demonstrated high vascularity in the AAM mass (5). The characteristic imaging of AAM on MRI is isointense relative to muscle on T1-weighted images and hyperintense on T2-weighted images (14).

The differential diagnosis was considered mainly to exclude other myxoid-containing tumors such as intramuscular myxoma, myxoid liposarcoma and malignant fibrous histocytoma of the extremities. The definitive diagnosis is histological since the non-specific imaging appearance of AAM did not distinguish it from other myxoid neoplasms.
Histopathological examination revealed an AAM of the thigh. Histologically, the tumor was characterized by spindle and stellate cells in a loose myxoid stroma with a prominent vascular component (2). Immunohistochemical staining of the tumor, particularly the reactivity for desmin, $\alpha$-smooth muscle actin, muscle-specific actin, vimentin, CD-34, S-100 protein, estrogen and progestin receptors, is essential for diagnosis $(12,15,16)$.

This study is the second reported case of femoral AAM. Heffernan et al (5) reported a mass without pain overlying the upper part of the right thigh over the tensor fascia latae muscle. Ultrasonography revealed a lobulated mass located in the subcutaneous fat of the right thigh. Naturally, there are certain differences between the two cases in terms of location, form and symptoms. A wide resection taking the underlying fascia and subcutaneous fat as the circumferential margin was successful and no recurrence occurred in the 6 months after surgery in this case. Although the patient in our case underwent marginal surgical excision, there was no evidence of local recurrence in the 18 months following surgery. The study showed no statistical difference in recurrences between patients with negative margins and patients with positive margins (9). Long-term follow-up is essential to aid in the timely identification of AAM recurrence.

In conclusion, we reported an uncommon case of AAM of the thigh. When sonography reveals a mass of the thigh with sharply demarcated borders and a heterogeneous echotexture, and weak blood flow is observed on CDUS, AAM should be considered. The mainstay of treatment for AAM of the thigh is surgical excison.

\section{References}

1. Steeper TA and Rosai J: Aggressive angiomyxoma of the female pelvis and perineum. Report of nine cases of a distinctive type of gynaecologic soft-tissue neoplasm. Am J Surg Pathol 7: 463-475, 1983.

2. Sylvester DC, Kortequee S, Moor JW, et al: Aggressive angiomyxoma of larynx: case report and literature review. J Laryngol Otol 124: 793-795, 2010.

3. Idrees MT, Hoch BL, Wang BY and Unger PD: Aggressive angiomyxoma of the male genital region. Report of 4 cases with immunohistochemical evaluation including hormone receptor status. Ann Diagn Pathol 10: 197-204, 2006.

4. Pai CY, Nieh S, Lee JC, et al: Aggressive angiomyxoma of supraclavicular fossa: a case report. Head Neck 30: 821-824, 2008.

5. Heffernan EJ, Hayes MM, Alkubaidan FO, et al: Aggressive angiomyxoma of the thigh: case report. Skeletal Radiol 37: 673-678, 2008.

6. Fine BA, Munoz AK, Litz CE, et al: Primary medical management of recurrent aggressive angiomyxoma of the vulva with a gonadotropin-releasing hormone agonist. Gynecol Oncol 81: 120-122, 2001.

7. Chihara Y, Fujimoto K, Takada S, et al: Aggressive angiomyxoma in the scrotum expressing androgen and progesterone receptors. Int J Urol 10: 672-675, 2003.

8. Dahiya K, Jain S, Duhan N, et al: Aggressive angiomyxoma of vulva and vagina: a series of three cases and review of literature. Arch Gynecol Obstet 283: 1145-1148, 2011.

9. Chan YM, Hon E, Ngai SW, et al: Aggressive angiomyxoma in females: is radical resection the only option? Acta Obstet Gynecol Scand 79: 216-220, 2000.

10. Nyan DCK and Pemberton J: Large aggressive angiomyxoma of the perineum and perineum and pelvis: an alternative approach. Diseases of the Colon and Rectum 41: 514-516, 1998.

11. Jingping Z and Chunfu Z: Clinical experiences on aggressive angiomyxoma in China (report of 93 cases). Int J Gynecol Cancer 20: 303-307, 2010. 
12. Hatano K, Tsujimoto Y, Ichimaru A, et al: Rare case of aggressive angiomyxoma presenting as a retrovesical tumor. Int J Urol 13: 1012-1014, 2006.

13. Nucci MR and Fletcher CD: Vulvovaginal soft tissue tumors: update and review. Histopathology 36: 97-108, 2000.

14. Wiser A, Korach J, Gotlieb W, et al: Importance of accurate preoperative diagnosis in the management of aggressive angiomyxoma: report of three cases and review of the literature. Abdom Imaging 31: 383-386, 2006.
15. Fetsch JF, Laskin WB, Lefkowitz M, et al: Aggressive angiomyxoma: a clinicopathological study of 29 female patients. Cancer 78: 79-90, 1996.

16. Begin LR, Clement PB, Kirk ME, et al: Aggressive angiomyxoma of pelvis soft parts: a clinicopathologic study of nine cases. Hum Pathol 16: 621-628, 1985. 